\title{
Simulation modeling for clinical trials evaluating radiotherapy omission in low-risk early breast cancer
}

\author{
Pierfrancesco Franco, Umberto Ricardi \\ Department of Oncology, Radiation Oncology, University of Turin, Turin, Italy \\ Correspondence to: Pierfrancesco Franco, MD, PhD. Department of Oncology, Radiation Oncology, University of Turin School of Medicine, Via \\ Genova 3, 10126 Turin, Italy. Email: pierfrancesco.franco@unito.it. \\ Comment on: Jayasekera J, Li Y, Schechter CB, et al. Simulation Modeling of Cancer Clinical Trials: Application to Omitting Radiotherapy in Low- \\ risk Breast Cancer. J Natl Cancer Inst 2018. [Epub ahead of print].
}

Submitted Aug 30, 2018. Accepted for publication Sep 27, 2018.

doi: $10.21037 / \mathrm{atm} .2018 .09 .63$

View this article at: http://dx.doi.org/10.21037/atm.2018.09.63

Adjuvant whole breast radiation therapy (WBI) is regarded as a standard approach for early-stage breast cancer (EBC) patients after breast conservation surgery (BCS) $(1,2)$. It is able to halve the 10 -year rate of any breast cancer recurrence (absolute reduction: 15.7\%) and to reduce by about $1 / 6^{\text {th }}$ the 15 -year breast cancer-related mortality (absolute reduction: 3.8\%) (3). However, if we take node negative EBC patients with low risk factors (tumors $<2 \mathrm{~cm}$, oestrogen receptor positive, older age, hormonal therapy given), the 10-year absolute decrease in the risk of recurrence is not negligible (around 10\%), but corresponds to an absolute reduction in the 15 -year risk of death due to breast cancer of $0.1 \%$ (3). Thus, in lowrisk $\mathrm{EBC}$, the benefit derived from combined modality treatment (including WBI) in terms of reduction in local recurrence rate and breast-cancer specific mortality may be overweighed by the burden of treatment-related acute and long-term toxicity (4). For this setting of patients, different approaches were set up to decrease the burden associated to radiation treatment including the use of hypofractionation, the selective employment of the boost to the lumpectomy cavity, accelerated partial breast irradiation and selective omission of WBI after BCS (5-9). Prospective trials investigated the hypothesis that radiotherapy omission after BCS would not be harmful to patients, but the correct selection of the population subset at 'low risk' is still open $(9,10)$. In this sense, new generation trials are presently ongoing to clarify this point, using either immunohistochemistry, genomic expression or signature assays (11). The study by Jayasekera et al. provides an interesting methodological perspective on how to deal with a clinical question employing a simulation approach (12). Evidence-based medicine is set upon the level of evidence of the available clinical data. Randomized controlled clinical trials are a pillar for high-level clinical evidence. They are based on a pre-established estimation of the parameter to be investigated in order to set a fixed type I error and a specific statistical power. The estimation of the parameter value can be statistically modelled and simulated to provide multiple outcome measures to finally obtain a probability distribution representing the investigated parameter (12). This methodology has been applied by the authors to the context of WBI omission after BCS in low-risk EBC. In the simulation, women enrolled were aged 49-74 with low- risk node-negative $(<2 \mathrm{~cm}$, oestrogen/progesterone receptor positive, human epidermal growth factor negative) breast cancer, having an Oncotype Dx score $\leq 18$. Primary endpoint was recurrence-free interval (RFI) (the time from randomization to any invasive recurrence or breast cancerrelated death). Secondary endpoints included loco-regional and distant RFI, breast-cancer-specific and overall survival. Non-inferiority of the experimental arm (WBI omission) would be concluded if the 2 -sided $90 \%$ confidence interval of the RFI hazard ratio (WBI omission vs. WBI) was $<1.7$ (5\% type I error and $80 \%$ power; 88 events required). The simulation predicted the need for 2,194 patients to be enrolled (in 40 months) with a subsequent 4.4 years observation time for a total trial duration of 9 years. Interestingly, the study introduced a selection criterion for patient's accrual based on biomarkers and specifically 
on a gene expression signature, able to stratify patients at low risk with the Oncotype DX (Genomic Health Inc., Redwood City, CA, USA) test, a 21-gene recurrence score assay used to correctly estimate the loco-regional recurrence risk in oestrogen receptor positive, node negative breast cancer patients (13). The same signature is being used within the IDEA (Individualized Decisions for Endocrine Therapy) trial, a multicentric prospective single-arm observational study evaluating the rate of locoregional relapse after BCS in post-menopausal women (age: 50-69 years) with tumor considered at low-risk, planned to be treated with post-operative endocrine therapy (14). The two independent models employed by Jayasekera et al. (based on different definitions of low-risk patients) concluded that the trial would be unlikely to demonstrate the noninferiority of WBI omission, which would conversely increase the rate of recurrence. This is in line with the recent meta-analysis by Chesney et al., evaluating data from 4 randomized controlled trials on radiotherapy omission in low-risk elderly patients (NSABP-B21, Toronto-British Columbia, CALGB 9343, PRIME II trials), which reported that the addition of radiotherapy to tamoxifen reduced the 5 -year breast recurrence from 60 to 10 per 1,000 patients (with an effect maintained at 10 years) and minimally the 5 -year axillary recurrence from 12 to 3 per 1,000 patients, with no effect on survival (15). The present study gives us some hints in dealing with the clinical scenario of omitting WBI in low-risk breast cancer patients. As pointed out by authors, it suggests that no identifiable threshold below which omitting WBI in non-inferior can be identified when a relative measure of radiotherapy effect (such as hazard ratio) is used (12). An absolute measure of efficacy would be preferable in this setting, with the possibility to avoid the need for randomization, given that the risk is sufficiently low (11). This is the methodological strategy employed in 4 out of 5 of the biomarker-based single-arm trials evaluating WBI omission in low-risk EBC (IDEA, PRECISION, LUMINA, PRIMETIME trials) (9). The outcome measure used by Jayasekera et al., RFI, is a reliable absolute measure of efficacy even if it equipoises local, regional and distant recurrences which may impact differently on patient's survival and quality of life. Another important point to be highlighted is that, since tolerability of treatment and patient's reported outcomes are important clinical endpoints, the real necessity of adjuvant systemic therapy in low-risk EBC needs to be explored. Hormonal therapy may be associated with a higher risk of osteoporotic complications and skeletal related events, sexual dysfunction, neurocognitive effects and cardiovascular disease (4). In adjunct, the benefit of endocrine therapy on overall survival in post-menopausal breast cancer patients still need to be demonstrated, while treatment compliance is low, given that only $35-60 \%$ of women are able to finalize a full 5-year adjuvant protocol (4). Overall, the side-effects of adjuvant systemic therapy are expected to outweigh those of WBRT, especially considering that technological advances in radiation therapy planning and delivery are considerably decreasing the global burden of treatment $(4,9)$. The appropriate allocation of low risk breast cancer patients to the most suitable treatment solution should be based not only on recurrence rate reduction and survival benefit, but also should include compliance to treatment, toxicity profile, quality of life, psychological and social aspects, as effectiveness and costs. Tumor biology should assist the clinician during the therapeutic decisional process. Validated multivariable endpoints and innovative tools for clinical assessment would be needed for correct treatment allocation. Modeling simulations study, such as the one by Jayasekera et al., would be extremely helpful to predict the outcome of future trials (and the uncertainty associated to the specific prediction), investigate key points including selection criteria and presumed effects of treatment and to better weight data derived from historical trials.

\section{Acknowledgements}

None.

\section{Footnote}

Conflicts of interest: The authors have no conflicts of interest to declare.

\section{References}

1. Veronesi U, Cascinelli N, Mariani L, et al. Twentyyear follow-up of a randomized study comparing breastconserving surgery with radical mastectomy for early breast cancer. N Engl J Med 2002;347:1227-32.

2. Franco $\mathrm{P}$, Zeverino $M$, Migliaccio F, et al. Intensitymodulated adjuvant whole breast radiation delivered with static angle tomotherapy (TomoDirect): a prospective case series. J Cancer Res Clin Oncol 2013;139:1927-36.

3. Early Breast Cancer Trialists' Collaborative Group (EBCTCG)., Darby S, McGale P, et al. Effect of radiotherapy after breast-conserving surgery on 10- 
year recurrence and 15-year breast cancer death: metaanalysis of individual patient data for 10,801 women in 17 randomised trials. Lancet 2011;378:1707-16.

4. Poortmans PMP, Arenas M, Livi L. Over-irradiation. Breast 2017;31:295-302.

5. Arcadipane F, Franco P, De Colle C, et al. Hypofractionation with no boost after breast conservation in early-stage breast cancer patients. Med Oncol 2016;33:108.

6. Rovea P, Fozza A, Franco P, et al. Once-Weekly Hypofractionated Whole-Breast Radiotherapy After Breast-Conserving Surgery in Older Patients: A Potential Alternative Treatment Schedule to Daily 3-Week Hypofractionation. Clin Breast Cancer 2015;15:270-6.

7. Livi L, Meattini I, Marrazzo L, et al. Accelerated partial breast irradiation using intensity-modulated radiotherapy versus whole breast irradiation: 5-year survival analysis of a phase 3 randomised controlled trial. Eur J Cancer 2015;51:451-63.

8. Franco P, Cante D, Sciacero P, et al. Tumor Bed Boost Integration during Whole Breast Radiotherapy: A Review of the Current Evidence. Breast Care (Basel) 2015;10:44-9.

9. Franco P, Iorio GC, Bartoncini S, et al. De-escalation of breast radiotherapy after conserving surgery in low-risk early breast cancer patients. Med Oncol 2018;35:62.

10. Franco P, Freedman GM, Ricardi U, et al. "Simplicity is

Cite this article as: Franco P, Ricardi U. Simulation modeling for clinical trials evaluating radiotherapy omission in low-risk early breast cancer. Ann Transl Med 2018;6(Suppl 1):S43. doi: 10.21037/atm.2018.09.63 complexity resolved": the case of postoperative radiation therapy after breast conservation. Transl Cancer Res 2016;5:S1336-9.

11. Bhattacharya IS, Kirby AM, et al. Can Interrogation of Tumour Characteristics Lead us to Safely Omit Adjuvant Radiotherapy in Patients with Early Breast Cancer? Clin Oncol (R Coll Radiol) 2018;30:158-65.

12. Jayasekera J, Li Y, Schechter CB, et al. Simulation Modeling of Cancer Clinical Trials: Application to Omitting Radiotherapy in Low-risk Breast Cancer. J Natl Cancer Inst 2018. [Epub ahead of print].

13. Mamounas EP, Tang G, Fisher B, et al. Association between the 21-gene recurrence score assay and risk of locoregional recurrence in node-negative, estrogen receptor-positive breast cancer: results from NSABP B-14 and NSABP B-20. J Clin Oncol 2010;28:1677-83.

14. The IDEA Study (Individualized Decisions for Endocrine Therapy Alone) at ClinicalTrials.gov; (as accessed March 10th 2018). Available online: https://clinicaltrials.gov/ct2/ show/NCT02400190

15. Chesney TR, Yin JX, Rajaee N, et al. Tamoxifen with radiotherapy compared with Tamoxifen alone in elderly women with early-stage breast cancer treated with breast conserving surgery: A systematic review and meta-analysis. Radiother Oncol 2017;123:1-9. 